\title{
Impact of predator fear on two competing prey species
}

\section{Debasis Mukherjee}

To cite this article:

D. Mukherjee,"Impact of predator fear on two competing prey species", Jambura J. Biomath, vol. 2, no. 1, pp. $1-12,2021$

DOI: $h$ ttps://doi.org/10.34312/jjbm.v2i1.9249

(C) 2021 Author(s).

\section{Articles You may be interested in}

Bifurkasi Hopf pada model prey-predator-super predator dengan fungsi respon yang berbeda

\section{Savitri and H. S. Panigoro}

http://dx.doi.org/10.34312/jjbm.v1i2.8399

Analisis kestabilan model predator-prey dengan infeksi penyakit pada prey dan pemanenan proporsional pada predator

S. Maisaroh, Resmawan, and E. Rahmi

http://dx.doi.org/10.34312/jjbm.v1i1.5948

Bifurkasi Hopf pada model Lotka-Volterra orde-fraksional dengan Efek Allee aditif pada predator

H. S. Panigoro and D. Savitri

http://dx.doi.org/10.34312/jjbm.v1i1.6908

Discrete-time prey-predator model with $\theta$-logistic growth for prey incorporating square root functional response

\section{P. K. Santra}

http://dx.doi.org/10.34312/jjbm.v1i2.7660

A stage-structure Rosenzweig-MacArthur model with effect of prey refuge

L. K. Beay and M. Saija

http://dx.doi.org/10.34312/jjbm.v1i1.6891 


\title{
Impact of predator fear on two competing prey species
}

\author{
Debasis Mukherjee \\ Department of Mathematics, Vivekananda College, Thakurpukur, Kolkata-700063, India \\ Corresponding author. Email: mukherjee1961@gmail.com
}

\begin{abstract}
Predator-prey interaction is a fundamental feature in the ecological system. The majority of studies have addressed how competition and predation affect species coexistence. Recent field studies on vertebrate has shown that fear of predators can influence the behavioural pattern of prey populations and reduce their reproduction. A natural question arises whether species coexistence is still possible or not when predator induce fear on competing species. Based on the above observation, we propose a mathematical model of two competing prey-one predator system with the cost of fear that affect not only the reproduction rate of both the prey population but also the predation rate of predator. To make the model more realistic, we incorporate intraspecific competition within the predator population. Biological justification of the model is shown through positivity and boundedness of solutions. Existence and stability of different boundary equilibria are discussed. Condition for the existence of coexistence equilibrium point is derived from showing uniform persistence. Local as well as a global stability criterion is developed. Bifurcation analysis is performed by choosing the fear effect as the bifurcation parameter of the model system. The nature of the limit cycle emerging through a Hopf bifurcation is indicated. Numerical experiments are carried out to test the theoretical results obtained from this model.
\end{abstract}

Keywords: Fear Effect; Predator-Prey Interaction; Stability; Bifurcation

\section{Introduction}

In ecological system, predation and competition are often assumed to be the important factors that affect species coexistence [1-4]. It is further investigated thoroughly in [5-7]. Gurevitch et al. [8] showed that predator can promote coexistence by lowering the strength of competition. It is a well known fact that predator can affect prey populations by direct killing. Recent field studies show that the indirect effect of predator species on prey species has major impact than direct killing [9-13]. Thus, it is reasonable to incorporate the fear effect in the model focussed on the role of predator regarding the coexistence of competing species. This type of mechanism can slow down the competition in respect of resource competition. Thus avoidance behaviour developed by fear usually stimulates coexistence provided prey partition resources, but not predators, whereas it weaken coexistence if prey partition predators but not resources. Zanette et al. [14] carried out experiments on song sparrows and observed $40 \%$ reduction in offspring production due to fear from the predator. With this fact in mind, Wang et al. [15] first developed the predator-prey model incorporating the cost of fear into prey reproduction. They found that the cost of fear has no impact in dynamical behavior when predation follows Holling type I response function, whereas it can stabilize the system by discarding periodic orbits considering Holling type II response function. Since then several studies are found in predator-prey models by introducing a fear component in prey reproduction. Wang and Zou [16] investigated a predator-prey model with the cost of fear and adaptive avoidance of predators and established that both strong adaption of adult prey and the large cost of fear induces destabilizing effect while large population of predators stabilize the system. Sasmal and Takeuchi [17] discussed the dynamics of a prey-predator model incorporating two facts: fear effect and group defense. Mondal et al. [18] analyzed the predator-prey model considering both the effects of fear and additional food and showed stability of equilibrium points and Hopf bifurcation. Zhang et al. [19] investigated the influence of anti- predator behavior due to fear of predators to a Holling type II prey-predator model allowing a prey refuge and demonstrated the global stability analysis of the equilibria of the model and showed Hopf bifurcation. Previous studies [15, 16, 18-22] are mainly confined in two species that cannot properly explain the fear effect when multiple species are present and these studies also ignore the effect of fear on predation rates. So present study attempts to investigate the predator fear which affects behavior of competing prey species and predation rates. This study also addresses the question of species coexistence.

Takeuchi and Adachi [23] studied the following two competing prey and one predator model in Lotka-Volterra 
form:

$$
\begin{aligned}
& \frac{d x_{1}}{d t}=x_{1}\left(r_{1}-x_{1}-\alpha x_{2}-\varepsilon y\right), \\
& \frac{d x_{2}}{d t}=x_{2}\left(r_{2}-\beta x_{1}-x_{2}-\mu y\right), \\
& \frac{d y}{d t}=y\left(-d+c \varepsilon x_{1}+c \mu x_{2}\right) .
\end{aligned}
$$

Here the variables $x_{1}$ and $x_{2}$ represent the densities of prey $y$ that of predator. $r_{1}$ and $r_{2}$ are the intrinsic growth rate of prey. $\alpha$ and $\beta$ are parameters representing competitive effects between two prey. $\varepsilon$ and $\mu$ are coefficients of decrease of prey species due to predation. $c$ is the equal conversion rate of the predator. All the parameters are assumed to be positive. In [23], the authors showed stability and Hopf bifurcation. They also pointed out that the stable equilibrium bifurcates to a periodic motion with a small amplitude when the predation rate increases and chaotic motion appears when one of two prey is superior than the other. Finally, they remarked that predator mediated coexistence is possible by the close relationship between preferences of a predator and competitive capacities of two prey. However, studies in [23] only considers the effect of direct killing prey populations and ignore the fear effect in the model equations. In the real world, the intraspecific competition among predator exists. Fear reduces the mobility of prey and reproduction so predation rates $\varepsilon$ and $\mu$ cannot be a constant, they are decreasing function of $k_{1}$ and $k_{2}$ respectively. By biological meaning, $f\left(k_{1}\right)$ and $g\left(k_{2}\right)$ are assumed to satisfy

$$
f^{\prime}\left(k_{1}\right)<0, f(0)=\varepsilon_{0}, \lim _{k_{1} \rightarrow \infty} f\left(k_{1}\right)=0, g^{\prime}\left(k_{2}\right)<0, g(0)=\mu_{0} \text { and } \lim _{k_{2} \rightarrow \infty} g\left(k_{2}\right)=0 .
$$

Taking the cost of fear on reproduction of prey and predation terms only, intraspecific competition among the predators and unequal conversion rate of predator, system (1) becomes

$$
\begin{aligned}
& \frac{d x_{1}}{d t}=x_{1}\left(\frac{r_{1}}{1+k_{1} y}-x_{1}-\alpha x_{2}-f\left(k_{1}\right) y\right), \\
& \frac{d x_{2}}{d t}=x_{2}\left(\frac{r_{2}}{1+k_{2} y}-\beta x_{1}-x_{2}-g\left(k_{2}\right) y\right), \\
& \frac{d y}{d t}=y\left(-d+c_{1} f\left(k_{1}\right) x_{1}+c_{2} g\left(k_{2}\right) x_{2}-h y\right),
\end{aligned}
$$

where $k_{i}, i=1,2$ represents the level of fear and $h$ denotes the intraspecific competition within the predator population. $c_{i}, i=1,2$ is the conversion efficiency of the predator. Justification for considering the fear term can be found in [15].

The rest of the paper is organized as follows. In Section 2, we study positivity and boundedness of the solutions of the system. In Section 3, existence and stability of different equilibrium points are discussed. Hopf bifurcation around the positive equilibrium point and the nature of the limit cycle emerging through Hopf bifurcation are derived in Section 4. Numerical simulations are performed in Section 5. A brief discussion concludes in Section 6.

\section{Positivity and Boundedness of Solutions}

In this section, we first show positivity and boundedness of solutions of system (2). These are very important so far as the biological validity of the model is concerned. We first establish the positivity.

Lemma 1. All solutions $\left(x_{1}(t), x_{2}(t), y(t)\right)$ of system (2) with initial values $\left(x_{10}, x_{20}, y_{0}\right) \in \mathbb{R}_{+}^{3}$, remains positive for all $t>0$.

proof. The positivity of $x_{1}(t), x_{2}(t), y(t)$ can be verified by the equations

$$
\begin{aligned}
& x_{1}(t)=x_{10} \exp \left\{\int_{0}^{t}\left[\frac{r_{1}}{1+k_{1} y(s)}-x_{1}(s)-\alpha x_{2}(s)-f\left(k_{1}\right) y(s)\right] d s\right\}, \\
& x_{2}(t)=x_{20} \exp \left\{\int_{0}^{t}\left[\frac{r_{2}}{1+k_{2} y(s)}-\beta x_{1}(s)-x_{2}(s)-g\left(k_{2}\right) y(s)\right] d s\right\}, \\
& y(t)=y_{0} \exp \left\{\int_{0}^{t}\left[-d+c_{1} f\left(k_{1}\right) x_{1}(s)+c_{2} g\left(k_{2}\right) x_{2}-h y(s)\right] d s\right\},
\end{aligned}
$$

with $x_{10}, x_{20}, y_{0}>0$. As $x_{10}>0$ then $x_{1}(t)>0$ for all $t>0$. The same argument is valid for component $x_{2}(t)$ and $y(t)$. Hence the interior of $\mathbb{R}_{+}^{3}$, is an invariant set of system (2). 
Lemma 2. All solutions of system (2) will lie in the region

$$
B=\left\{\left(x_{1}, x_{2}, y\right) \in \mathbb{R}_{+}^{3}: 0 \leq c_{1} x_{1}+c_{2} x_{2}+y \leq \frac{M}{\lambda}\right\}
$$

as $t \rightarrow \infty$ for all positive initial values $\left(x_{10}, x_{20}, y_{0}\right) \in \mathbb{R}_{+}^{3}$, where $\lambda<\min \left\{r_{1}, r_{2}, d\right\}$ and $M=r_{1}^{2}+r_{2}^{2}$.

proof. Let us consider the function

$$
W(t)=c_{1} x_{1}+c_{2} x_{2}+y .
$$

The time derivative along a solution of (2) is

$$
\frac{d W(t)}{d t}=c_{1} x_{1}\left(\frac{r_{1}}{1+k_{1} y}-x_{1}-\alpha x_{2}\right)+c_{2} x_{2}\left(\frac{r_{2}}{1+k_{2} y}-\beta x_{1}-x_{2}\right)-y(d+h y) .
$$

For each $\lambda>0$, the following inequality is satisfied.

$$
\begin{aligned}
\frac{d W}{d t}+\lambda W & \leq c_{1} x_{1}\left(r_{1}+\lambda-x_{1}\right)+c_{2} x_{2}\left(r_{2}+\lambda-x_{2}\right)+(\lambda-d) y \\
& =-\left\{\left(x_{1}-\frac{r_{1}+\lambda}{2}\right)^{2}-\left(\frac{r_{1}+\lambda}{2}\right)^{2}\right\}-\left\{\left(x_{2}-\frac{r_{2}+\lambda}{2}\right)^{2}-\left(\frac{r_{2}+\lambda}{2}\right)^{2}\right\}+(\lambda-d) y \\
& \leq \frac{\left(r_{1}+\lambda\right)^{2}}{4}+\frac{\left(r_{2}+\lambda\right)^{2}}{4}+(\lambda-d) y
\end{aligned}
$$

Now choose $\lambda$ such that $\left.\lambda<\min \left\{r_{1}, r_{2}, d\right\}\right\}$. Thus (3) can be written as

$$
\frac{d W}{d t}+\lambda W \leq r_{1}^{2}+r_{2}^{2}=M(\text { say })
$$

By using the comparison theorem [24], we get

$$
0 \leq W\left(x_{1}(t), x_{2}(t), y(t)\right) \leq \frac{M}{\lambda}+W\left(x_{1}(0), x_{2}(0), y(0)\right) / e^{\lambda t} .
$$

Taking limit when $t \rightarrow \infty$, we have $0<W(t) \leq \frac{M}{\lambda}$. Hence system (2) is bounded.

From the above Lemma 2 , we can find that $y(t) \leq \frac{M}{\lambda}=\bar{M}$ (say).

\section{Existence of equilibria and Stability analysis}

Evidently, system (2) has six non-negative equilibrium points. The population free equilibrium point $E_{0}=(0,0,0)$. The second prey and predator free equilibrium point $E_{1}=\left(r_{1}, 0,0\right)$. The first prey and predator free equilibrium point $E_{2}=\left(0, r_{2}, 0\right)$. If $\alpha<\frac{r_{1}}{r_{2}}<\frac{1}{\beta}$ or $\alpha>\frac{r_{1}}{r_{2}}>\frac{1}{\beta}$ then there exists unique predator free equilibrium point $E_{12}=\left(\bar{x}_{1}, \bar{x}_{2}, 0\right)$ where $\bar{x}_{1}=\frac{r_{1}-r_{2} \alpha}{1-\alpha \beta}, \bar{x}_{2}=\frac{r_{2}-r_{1} \beta}{1-\alpha \beta}$. If $d<r_{1} c_{1} f\left(k_{1}\right)$ then there exists unique second prey free equilibrium point $E_{13}=\left(\hat{x}_{1}, 0, \hat{y}\right)$ where $\hat{x}_{1}=\frac{h \hat{y}+d}{c_{1} f\left(k_{1}\right)}$ and $\hat{y}$ is the positive root of the equation

$$
k_{1}\left(h+c_{1} f\left(k_{1}\right)^{2}\right) y^{2}+\left(h+d k_{1}+c_{1} f\left(k_{1}\right)^{2}\right) y+d-r_{1} c_{1} f\left(k_{1}\right)=0
$$

If $d<r_{2} c_{2} g\left(k_{2}\right)$ then there exists unique first prey free equilibrium point $E_{23}=\left(0, \tilde{x}_{2}, \tilde{y}\right)$ where $\tilde{x}_{2}=\frac{h \tilde{y}+d}{c_{2} g\left(k_{2}\right)}$ and $\tilde{y}$ is the positive root of the equation

$$
k_{2}\left(h+c_{2} g\left(k_{2}\right)^{2}\right) y^{2}+\left(h+d k_{2}+c_{2} g\left(k_{2}\right)^{2}\right) y+d-r_{2} c_{2} g\left(k_{2}\right)=0 .
$$

Theorem 3. (i) $E_{0}$ is always unstable. 
(ii) $E_{1}$ is locally stable if $r_{2}<\beta r_{1}$ and $d>c_{1} f\left(k_{1}\right) r_{1}$.

(iii) $E_{2}$ is stable if $r_{1}<\alpha r_{2}$ and $d>c_{2} g\left(k_{2}\right) r_{2}$.

(iv) $E_{12}$ is stable if $\alpha \beta<1$ and $d>c_{1} f\left(k_{1}\right) \bar{x}_{1}+c_{2} g\left(k_{2}\right) \bar{x}_{2}$.

(v) $E_{13}$ is stable if $\frac{r_{2}}{1+k_{2} \hat{y}}<\beta \hat{x}_{1}+g\left(k_{2}\right) \hat{y}$ and $E_{23}$ is stable if $\frac{r_{1}}{1+k_{1} \tilde{y}}<\alpha \tilde{x}_{2}+f\left(k_{1}\right) \tilde{y}$.

proof. Proof follows immediately by linearizing around the equilibria.

To find the existence condition of positive equilibrium point, we first show uniform persistence of system (2) and then application of a result in [25] ensures the existence.

\section{Persistence}

In biological sense, persistence means the long term survival of all populations whatever may be the initial populations. Geometrically, it means the existence of a region in the phase space at a non-zero distance from the boundary in which all species enter and must lie ultimately.

Now we state a result establishing the uniform persistence of system (2).

Theorem 4. Suppose $E_{12}, E_{13}$ and $E_{23}$ exist. Further suppose that $d<c_{1} f\left(k_{1}\right) \bar{x}_{1}+c_{2} g\left(k_{2}\right) \bar{x}_{2}, \frac{r_{2}}{1+k_{2} \hat{y}}>\beta \hat{x}_{1}+g\left(k_{2}\right) \hat{y}$ and $\frac{r_{1}}{1+k_{1} \tilde{y}}>\alpha \tilde{x}_{2}+f\left(k_{1}\right) \tilde{y}$ then system (2) is uniformly persistent.

proof. We shall prove the theorem by using the idea of average Lyapunov function [26].

Consider the average Lyapunov function of the form : $H(x)=x_{1}{ }^{m_{1}} x_{2}{ }^{m_{2}} y^{m_{3}}$, where each $m_{i}, i=1,2,3$ is assumed positive. In the interior of $\mathbb{R}_{+}^{3}$, one has

$$
\begin{aligned}
\frac{1}{H(x)} \frac{d H(x)}{d t}= & \psi(x)=\frac{m_{1}}{x_{1}} \frac{d x_{1}}{d t}+\frac{m_{2}}{x_{2}} \frac{d x_{2}}{d t}+\frac{m_{3}}{y} \frac{d y}{d t} \\
= & m_{1}\left(\frac{r_{1}}{1+k_{1} y}-x_{1}-\alpha x_{2}-f\left(k_{1}\right) y\right)+m_{2}\left(\frac{r_{2}}{1+k_{2} y}-\beta x_{1}-x_{2}-g\left(k_{2}\right) y\right) \\
& +m_{3}\left(-d+c_{1} f\left(k_{1}\right) x_{1}+c_{2} g\left(k_{2}\right) x_{2}-h y\right)
\end{aligned}
$$

We have to show $\psi(x)>0$ for all $x \in b d \mathbb{R}_{+}^{3}$, for a suitable choice of $m_{1}, m_{2}, m_{3}>0$, to prove uniform persistence of system (2). That is one has to satisfy the following conditions corresponding to the boundary equilibria $E_{0}, E_{1}$, $E_{2}, E_{12}, E_{13}, E_{23}$ only as there are no periodic orbits in $x_{1}-x_{2}, x_{1}-y$ and $x_{2}-y$ plane.

$$
\begin{aligned}
E_{0} & : m_{1} r_{1}+m_{2} r_{2}-m_{3} d>0 \\
E_{1} & : m_{2}\left(r_{2}-\beta r_{1}\right)+m_{3}\left(-d+c_{1} f\left(k_{1}\right) r_{1}\right)>0 \\
E_{2} & : m_{1}\left(r_{1}-\alpha r_{2}\right)+m_{3}\left(-d+c_{2} g\left(k_{2}\right) r_{2}\right)>0 \\
E_{12} & : m_{3}\left(c_{1} f\left(k_{1}\right) \bar{x}_{1}+c_{2} g\left(k_{2}\right) \bar{x}_{2}-d\right)>0 \\
E_{13} & : m_{2}\left(\frac{r_{2}}{1+k_{2} \hat{y}}-\beta \hat{x}_{1}-g\left(k_{2}\right) \hat{y}\right)>0 \\
E_{23} & : m_{1}\left(\frac{r_{1}}{1+k_{1} \tilde{y}}-\alpha \tilde{x}_{2}-f\left(k_{1}\right) \tilde{y}\right)>0
\end{aligned}
$$

Since $d<c_{1} f\left(k_{1}\right) \bar{x}_{1}+c_{2} g\left(k_{2}\right) \bar{x}_{2}, \frac{r_{2}}{1+k_{2} \hat{y}}>\beta \hat{x}_{1}+g\left(k_{2}\right) \hat{y}$ and $\frac{r_{1}}{1+k_{1} \tilde{y}}>\alpha \tilde{x}_{2}+f\left(k_{1}\right) \tilde{y}$ positivity of (7), (8) and (9) is obvious. Again existence of $E_{13}$ and $E_{23}$ implies that $d<c_{1} f\left(k_{1}\right) r_{1}$ and $c_{2} g\left(k_{2}\right) r_{2}$. So positivity of (4), (5) and (6) will follow by the suitable choice of $m_{1}, m_{2}$, and $m_{3}$. This completes the proof.

In this section, we proved that system (2) ensures uniform persistence provided that the conditions of Theorem 4 are satisfied. Further, it is proved in [25], uniform persistence implies the existence of an interior equilibrium point. Hence $E^{*}=\left(x_{1}^{*}, x_{2}^{*}, y^{*}\right)$ exists; that is in effect Theorem 4 implies that $E^{*}$ exists. 
Theorem 5. Suppose all the conditions of Theorem 4 be satisfied. Then the interior equilibrium point $E^{*}$ of system (2) is locally asymptotically stable if $\alpha<\frac{c_{2} g\left(k_{2}\right)}{c_{1} f\left(k_{1}\right)}<\frac{1}{\beta}$.

proof. The Jacobian matrix of system (2) for the equilibrium point $E^{*}$ is given by

$$
J\left(E^{*}\right)=\left(\begin{array}{ccc}
-x_{1}^{*} & -\alpha x_{1}^{*} & -\left(f\left(k_{1}\right)+\frac{r_{1} k_{1}}{\left(1+k_{1} y^{*}\right)^{2}}\right) x_{1}^{*} \\
-\beta x_{2}^{*} & -x_{2}^{*} & -\left(g\left(k_{2}\right)+\frac{r_{2} k_{2}}{\left(1+k_{2} y^{*}\right)^{2}}\right) x_{2}^{*} \\
c_{1} f\left(k_{1}\right) y^{*} & c_{2} g\left(k_{2}\right) y^{*} & -h y^{*}
\end{array}\right) .
$$

The characteristic equation about $E^{*}$ is

$$
\lambda^{3}+a_{1} \lambda^{2}+a_{2} \lambda+a_{3}=0
$$

where

$$
\begin{aligned}
a_{1}= & x_{1}^{*}+x_{2}^{*}+h y^{*}, \\
a_{2}= & x_{1}^{*} x_{2}^{*}(1-\alpha \beta)+h y^{*}\left(x_{1}^{*}+x_{2}^{*}\right)+\left(\frac{r_{1} k_{1}}{\left(1+k_{1} y^{*}\right)^{2}}+f\left(k_{1}\right)\right) c_{1} f\left(k_{1}\right) x_{1}^{*} y^{*} \\
& +\left(\frac{r_{2} k_{2}}{\left(1+k_{2} y^{*}\right)^{2}}+g\left(k_{2}\right)\right) c_{2} g\left(k_{2}\right) x_{2}^{*} y^{*}, \\
a_{3}= & x_{1}^{*} x_{2}^{*} y^{*}\left\{h(1-\alpha \beta)+\left(c_{2} g\left(k_{2}\right)-\alpha c_{1} f\left(k_{1}\right)\right)\left(\frac{r_{2} k_{2}}{\left(1+k_{2} y^{*}\right)^{2}}+g\left(k_{2}\right)\right)\right. \\
& \left.+\left(c_{1} f\left(k_{1}\right)-\beta c_{2} g\left(k_{2}\right)\right)\left(\frac{r_{1} k_{1}}{\left(1+k_{1} y^{*}\right)^{2}}+f\left(k_{1}\right)\right)\right\} .
\end{aligned}
$$

Clearly $a_{1}>0$. Again $a_{2}>0, a_{3}>0$, and $a_{1} a_{2}>a_{3}$ follows from the assumption of the theorem. Therefore the result holds by the application of Routh-Hurwitz criterion. This completes the proof.

Theorem 6. Suppose that $\left(\frac{\alpha+\beta}{2}\right)^{2}+\frac{1}{4 h}\left(c_{1}^{2} r_{1}^{2} k_{1}^{2}+c_{2}^{2} r_{2}^{2} k_{2}^{2}+\frac{1}{2}(\alpha+\beta) c_{1} r_{1} k_{1} c_{2} r_{2} k_{2}\right\}<1$ Then E* is globally asymptotically stable.

proof. Consider the following positive definite function about $E^{*}$.

$$
V(t)=c_{1}\left(x_{1}-x_{1}^{*}-x_{1}^{*} \ln \frac{x_{1}}{x_{1}^{*}}\right)+c_{2}\left(x_{2}-x_{2}^{*}-x_{2}^{*} \ln \frac{x_{2}}{x_{2}^{*}}\right)+\left(y-y^{*}-y^{*} \ln \frac{y}{y^{*}}\right) .
$$

Differentiating $V$ with respect to $t$ along the solution of system (2), we get

$$
\begin{aligned}
\frac{d V}{d t}= & c_{1}\left(x_{1}-x_{1}^{*}\right)\left\{\frac{r_{1}}{1+k_{1} y}-x_{1}-\alpha x_{2}-f\left(k_{1}\right) y\right\}+c_{2}\left(x_{2}-x_{2}^{*}\right)\left(\frac{r_{2}}{1+k_{2} y}-\beta x_{1}-x_{2}-g\left(k_{2}\right) y\right) \\
& +\left(y-y^{*}\right)\left(-d+c_{1} f\left(k_{1}\right) x_{1}+c_{2} g\left(k_{2}\right) x_{2}-h y\right) \\
= & c_{1}\left(x_{1}-x_{1}^{*}\right)\left\{\frac{r_{1} k_{1}\left(y^{*}-y\right)}{\left(1+k_{1} y\right)\left(1+k_{1} y^{*}\right)}-\left(x_{1}-x_{1}^{*}\right)-\alpha\left(x_{2}-x_{2}^{*}\right)\right\} \\
& -c_{2}\left(x_{2}-x_{2}^{*}\right)\left\{\frac{r_{2} k_{2}\left(y^{*}-y\right)}{\left(1+k_{2} y\right)\left(1+k_{2} y^{*}\right)}-\beta\left(x_{1}-x_{1}^{*}\right)-\left(x_{2}-x_{2}^{*}\right)\right\}-h\left(y-y^{*}\right)^{2} \\
\leq & -\left(x_{1}-x_{1}^{*}\right)^{2}+(\alpha+\beta)\left|\left(x_{1}-x_{1}^{*}\right)\right|\left|\left(x_{2}-x_{2}^{*}\right)\right|-\left(x_{2}-x_{2}^{*}\right)^{2}-h\left(y-y^{*}\right)^{2} \\
& +c_{1} r_{1} k_{1}\left|x_{1}-x_{1}^{*}\right|\left|y-y^{*}\right|+c_{2} r_{2} k_{2}\left|x_{2}-x_{2}^{*}\right|\left|y-y^{*}\right|
\end{aligned}
$$

Clearly $\dot{V}$ is negative definite if the following matrix $A$ is positive definite.

$$
A=\left(\begin{array}{ccc}
1 & -\frac{1}{2}(\alpha+\beta) & -\frac{1}{2} c_{1} r_{1} k_{1} \\
-\frac{1}{2}(\alpha+\beta) & 1 & -\frac{1}{2} c_{2} r_{2} k_{2} \\
-\frac{1}{2} c_{1} r_{1} k_{1} & -\frac{1}{2} c_{2} r_{2} c_{2} & h
\end{array}\right)
$$


Thus the condition of theorem implies that $A$ is positive and consequently $V$ is a Lyapunov function and hence the theorem follows.

\section{Hopf bifurcation and its nature}

Set $F\left(k_{1}\right)=a_{1}\left(k_{1}\right) a_{2}\left(k_{1}\right)-a_{3}\left(k_{1}\right)$.

Theorem 7. Assume that $a_{3}\left(k_{1}\right)>0$. If there exists $k_{1}=k_{1}^{*}$ such that (i) $F\left(k_{1}^{*}\right)=0$, (ii) $F^{\prime}\left(k_{1}^{*}\right)>0$ then the positive equilibrium point $E^{*}$ is unstable if $k_{1}<k_{1}^{*}$ but is stable for $k_{1}>k_{1}^{*}$ and a Hopf bifurcation of periodic solution occurs at $k_{1}=k_{1}^{*}$.

proof. Proceeding along the lines in [27], we note that the condition $F^{\prime}\left(k_{1}^{*}\right)>0$ indicates that $F\left(k_{1}\right)$ is monotonic increasing function in the neighbourhood of $k_{1}=k_{1}^{*}$. Now, $F\left(k_{1}\right)<F\left(k_{1}^{*}\right)=0$ for $k_{1}<k_{1}^{*}$ this implies that $a_{1}\left(k_{1}\right) a_{2}\left(k_{1}\right)-a_{3}\left(k_{1}\right)<0$ and hence the condition of Routh-Hurwitz criterion is violated. Consequently, $E^{*}$ becomes unstable. Again, it is obvious that, $F\left(k_{1}\right)>F\left(k_{1}^{*}\right)=0$ for $k_{1}>k_{1}^{*}$ and this implies that $a_{1}\left(k_{1}\right) a_{2}\left(k_{1}\right)-$ $a_{3}\left(k_{1}\right)>0$. Since $a_{1}\left(k_{1}\right)$ is always positive and $a_{3}\left(k_{1}\right)$ is positive by the assumption of the theorem. Thus we have $a_{2}\left(k_{1}\right)>0$. Therefore all the conditions of Routh-Hurwitz criterion are satisfied and hence $E^{*}$ is stable. Therefore, Hopf bifurcation follows from a result in [28].

\subsection{Stability of the limit cycle}

Stability of the limit cycle can be derived by calculating the coefficient of curvature of the limit cycle [29]. The aim of this section is to investigate the stability of limit cycle of system (2). We now shift the equilibrium point of system (2) from $\left(x_{1}^{*}, x_{2}^{*}, y^{*}\right)$ to $(0,0,0)$ through the following transformation

$$
x_{1}=u_{1}+x_{1}^{*}, x_{2}=u_{2}+x_{2}^{*}, y=u_{3}+y^{*} .
$$

In terms of the new variables, system (2) can be transformed in the following form :

$$
\begin{aligned}
\frac{d u_{1}}{d t} & =\frac{r_{1} u_{1}}{1+k_{1}\left(u_{3}+y^{*}\right)}-\left(u_{1}+x_{1}^{*}\right)^{2}-\alpha\left(u_{1}+x_{1}^{*}\right)\left(u_{2}+x_{2}^{*}\right)-f\left(k_{1}\right)\left(u_{1}+x_{1}^{*}\right)\left(u_{3}+y^{*}\right)+\frac{r_{1} x_{1}^{*}}{1+k_{1}\left(u_{3}+y^{*}\right)^{\prime}} \\
\frac{d u_{2}}{d t} & =\frac{r_{2} u_{2}}{1+k_{2}\left(u_{3}+y^{*}\right)}-\beta\left(u_{1}+x_{1}^{*}\right)\left(u_{2}+x_{2}^{*}\right)-\left(u_{2}+x_{2}^{*}\right)^{2}-g\left(k_{2}\right)\left(u_{2}+x_{2}^{*}\right)\left(u_{3}+y^{*}\right)+\frac{r_{2} x_{2}^{*}}{1+k_{2}\left(u_{3}+y^{*}\right)^{\prime}}, \\
\frac{d u_{3}}{d t} & =\left(u_{3}+y^{*}\right)\left(c_{1} f\left(k_{1}\right) u_{1}+c_{2} g\left(k_{2}\right) u_{2}-h u_{3}\right),
\end{aligned}
$$

where the matrix of the nonlinear part is

$$
Q=\left(\begin{array}{c}
Q_{1} \\
Q_{2} \\
Q_{3}
\end{array}\right)=\left(\begin{array}{r}
\frac{r_{1} u_{1}}{1+k_{1}\left(u_{3}+y^{*}\right)}-u_{1}^{2}-\alpha u_{1} u_{2}-f\left(k_{1}\right) u_{1} u_{3}+\frac{r_{1} x_{1}^{*}}{1+k_{1}\left(u_{3}+y^{*}\right)} \\
\frac{r_{2} u_{2}}{1+k_{2}\left(u_{3}+y^{*}\right)}-\beta u_{1} u_{2}-u_{2}^{2}-g\left(k_{2}\right) u_{2} u_{3}+\frac{r_{2} x_{2}^{*}}{1+k_{2}\left(u_{3}+y^{*}\right)} \\
c_{1} f\left(k_{1}\right) u_{1} u_{3}+c_{2} g\left(k_{2}\right) u_{2} u_{3}-h u_{3}^{2}
\end{array}\right)
$$

From the nonlinear part above, we calculate the characteristic quantities in the followings :

$$
\begin{aligned}
& g_{20}^{0}=\frac{1}{4}\left\{\frac{\partial^{2} Q_{1}}{\partial u_{1}^{2}}-\frac{\partial^{2} Q_{1}}{\partial u_{2}^{2}}+2 \frac{\partial^{2} Q_{2}}{\partial u_{1} \partial u_{2}}+i\left(\frac{\partial^{2} Q_{2}}{\partial u_{1}^{2}}-\frac{\partial^{2} Q_{2}}{\partial u_{2}^{2}}-2 \frac{\partial^{2} Q_{1}}{\partial u_{1} \partial u_{2}}\right)\right\}=-\frac{1}{2}\{(1+\beta)-i(1+\alpha)\}, \\
& g_{11}^{0}=\frac{1}{4}\left\{\frac{\partial^{2} Q_{1}}{\partial u_{1}^{2}}+\frac{\partial^{2} Q_{1}}{\partial u_{2}^{2}}+i\left(\frac{\partial^{2} Q_{2}}{\partial u_{1}^{2}}+\frac{\partial^{2} Q_{2}}{\partial u_{2}^{2}}\right)\right\}=-\frac{1}{2}\{1+i\} \\
& G_{110}^{0}=\frac{1}{2}\left\{\frac{\partial^{2} Q_{1}}{\partial u_{1} \partial u_{3}}+\frac{\partial^{2} Q_{2}}{\partial u_{2} \partial u_{3}}+i\left(\frac{\partial^{2} Q_{2}}{\partial u_{1} \partial u_{3}}-\frac{\partial^{2} Q_{1}}{\partial u_{2} \partial u_{3}}\right)\right\}=-\frac{1}{2}\left\{\frac{r_{1} k_{1}}{\left\{1+k_{1}\left(u_{3}+y^{*}\right)\right\}^{2}}+f\left(k_{1}\right)+g\left(k_{2}\right)\right\}, \\
& G_{101}^{0}=\frac{1}{2}\left\{\frac{\partial^{2} Q_{1}}{\partial u_{1} \partial u_{3}}-\frac{\partial^{2} Q_{2}}{\partial u_{2} \partial u_{3}}+i\left(\frac{\partial^{2} Q_{2}}{\partial u_{1} \partial u_{3}}+\frac{\partial^{2} Q_{1}}{\partial u_{2} \partial u_{3}}\right)\right\}=-\frac{1}{2}\left[\frac{r_{1}}{\left\{1+k_{1}\left(u_{3}+y^{*}\right)\right\}^{2}}+f\left(k_{1}\right)-g\left(k_{2}\right)\right], \\
& W_{11}^{0}=-\frac{1}{4 \lambda_{3}\left(a_{1}\left(h^{*}\right)\right)}\left(\frac{\partial^{2} Q_{3}}{\partial u_{1}^{2}}+\frac{\partial^{2} Q_{3}}{\partial u_{2}^{2}}\right)=0,
\end{aligned}
$$




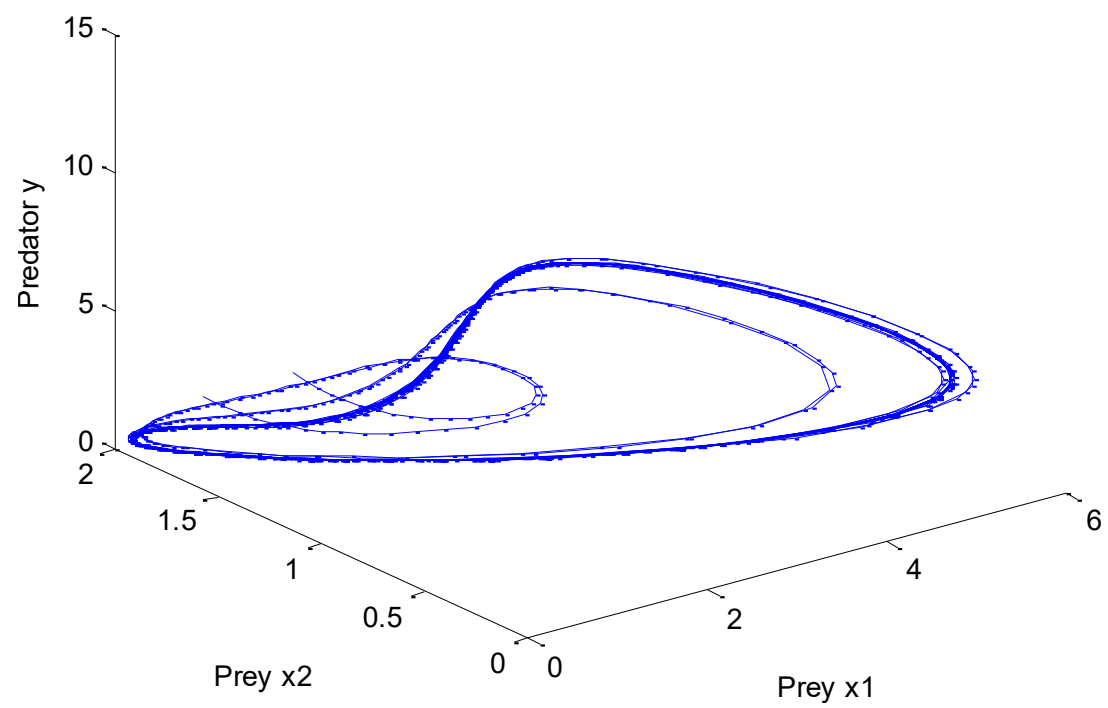

Figure 1. Phase portrait of system (11) without fear and the other parameter values are defined in (12)

$$
\begin{aligned}
& W_{20}^{0}=-\frac{1}{4\left(4 i-\lambda_{3}\left(a_{1}\left(h^{*}\right)\right)\right.}\left(\frac{\partial^{2} Q_{3}}{\partial u_{1}^{2}}-\frac{\partial^{2} Q_{3}}{\partial u_{2}^{2}}-2 i \frac{\partial^{2} Q_{3}}{\partial u_{1} \partial u_{2}}\right)=0 \\
& G_{21}^{0}=\frac{1}{8}\left\{\frac{\partial^{3} Q_{1}}{\partial u_{1}^{3}}+\frac{\partial^{3} Q_{1}}{\partial u_{1} \partial u_{2}^{2}}+\frac{\partial^{3} Q_{2}}{\partial u_{2}^{3}}+\frac{\partial^{3} Q_{2}}{\partial u_{1}^{2} \partial u_{2}}+i\left(\frac{\partial^{3} Q_{2}}{\partial u_{1}^{3}}+\frac{\partial^{3} Q_{2}}{\partial u_{1} \partial u_{2}^{2}}-\frac{\partial^{3} Q_{1}}{\partial u_{1}^{2} \partial u_{2}}-\frac{\partial^{3} Q_{1}}{\partial u_{2}^{3}}\right)\right\}=0 .
\end{aligned}
$$

Then the coefficient of curvature of limit cycle of system (2) is

$$
\begin{aligned}
\sigma_{1}^{0} & =\operatorname{Re}\left\{\frac{g_{20}^{0} g_{11}^{0}}{4} i+G_{110}^{0} W_{11}^{0}+\frac{G_{21}^{0}+G_{101}^{0} W_{20}^{0}}{2}\right\} \\
& =\frac{1}{16}(\alpha-\beta) .
\end{aligned}
$$

Thus we observed that the coefficient of curvature $\sigma_{1}^{0}<0$ if $\alpha<\beta$ in that case the limit cycle of system (2) will be stable. From above analysis one can conclude that the interspecific competition rate between the prey species plays a vital role for determining the nature of the limit cycle emerging through Hopf bifurcation.

In the following table, we summarise the stability criteria of different equilibria of system (2).

Table 1. Dynamics of system (2). LAS= Locally asymptotically stable, GAS= Globally asymptotically stable.

\begin{tabular}{|c|c|c|}
\hline Equilibria & Stability condition & Equilibrium nature \\
\hline$E_{0}$ & No condition & Unstable \\
$E_{1}$ & $\frac{r_{2}}{\beta}<r_{1}<\frac{d}{c_{1} f\left(k_{1}\right)}$ & LAS \\
$E_{2}$ & $\frac{r_{1}}{\alpha}<r_{2}<\frac{d}{c_{2} g\left(k_{2}\right)}$ & LAS \\
$E_{12}$ & $\alpha \beta<1, \frac{d}{r_{2}}>c_{1} f\left(k_{1}\right) \bar{x}_{1}+c_{2} g\left(k_{2}\right) \bar{x}_{2}$ & LAS \\
$E_{13}$ & $\frac{r_{1}}{1+k_{2} \hat{y}}<\beta \hat{x}_{1}+g\left(k_{2}\right) \hat{y}$ & LAS \\
$E_{23}$ & $\frac{r_{1}}{1+k_{1} \tilde{y}}<\alpha \tilde{x}_{2}+f\left(k_{1}\right) \tilde{y}$ & LAS \\
$E^{*}$ & $\alpha<\frac{c_{2} g\left(k_{2}\right)}{c_{1} f\left(k_{1}\right)}<\frac{1}{\beta}$ & LAS \\
$E^{*}$ & $\left(\frac{\alpha+\beta}{2}\right)^{2}+\frac{1}{4 h}\left(c_{1}^{2} r_{1}^{2} k_{1}^{2}+c_{2}^{2} r_{2}^{2} k_{2}^{2}+\frac{1}{2}(\alpha+\beta) c_{1} r_{1} k_{1} c_{2} r_{2} k_{2}\right\}<1$ & GAS \\
\hline
\end{tabular}




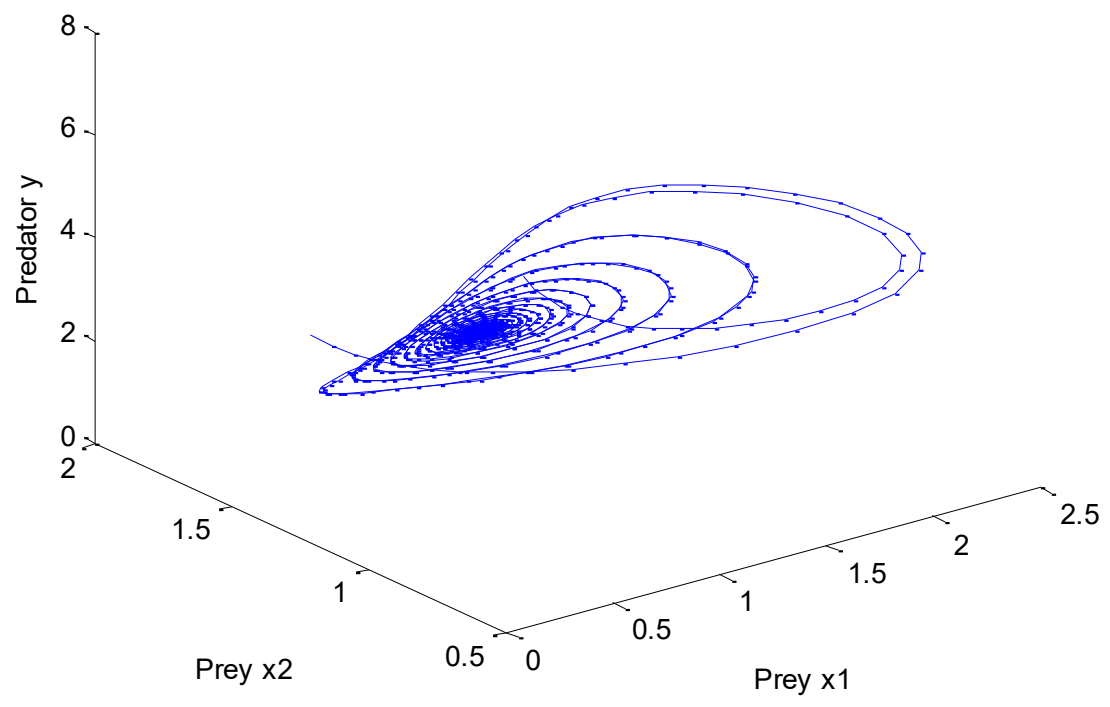

Figure 2. Phase portrait of system (11) for with parameters values (12) and $k_{1}=0, k_{2}=0.04$

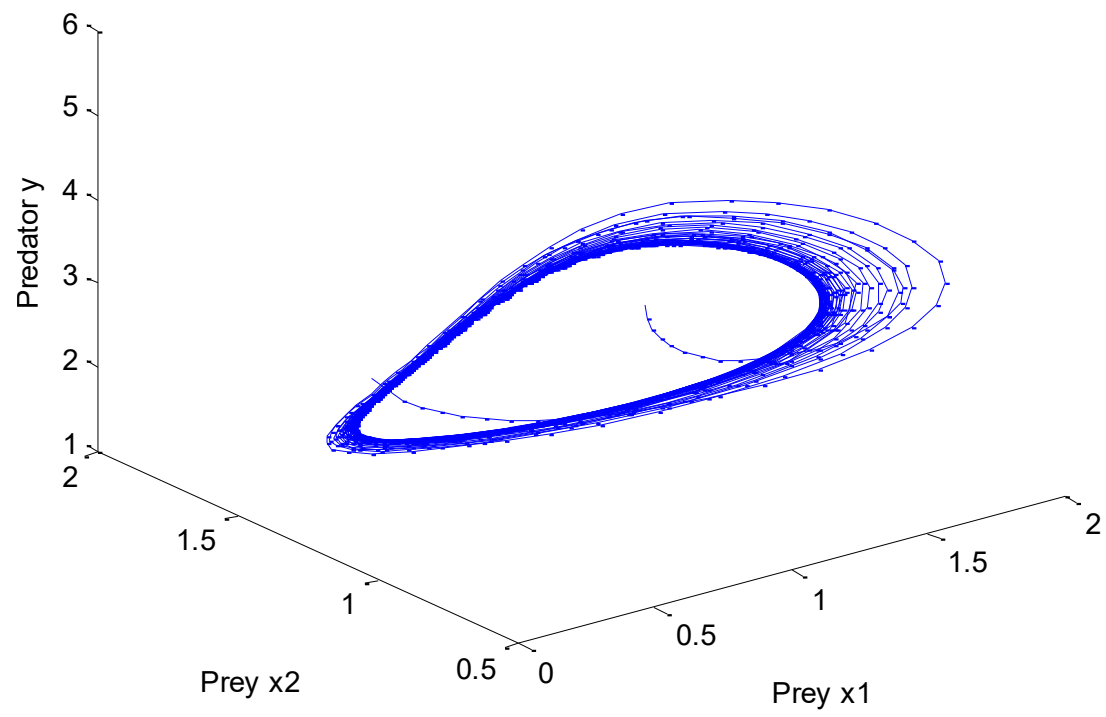

Figure 3. Phase portrait of system (11) for with parameters values (12) and $k_{1}=0.05, k_{2}=0.04$ showing stable limit cycle surrounding $E^{*}=(1.5347,0.9467,2.7008)$

\section{Numerical Simulations}

In this section, we present some numerical simulations to illustrate the analytical results obtained earlier. For this purpose, we choose a particular form of the functions $f\left(k_{1}\right)$ and $g\left(k_{2}\right)$ given by

$$
f\left(k_{1}\right)=\frac{\varepsilon_{0}}{1+k_{1} m}, g\left(k_{2}\right)=\frac{\mu_{0}}{1+k_{2} n}
$$

and these lead (2) to the following system:

$$
\begin{aligned}
& \frac{d x_{1}}{d t}=x_{1}\left(\frac{r_{1}}{1+k_{1} y}-x_{1}-\alpha x_{2}-\frac{\varepsilon_{0}}{1+k_{1} m} y\right), \\
& \frac{d x_{2}}{d t}=x_{2}\left(\frac{r_{2}}{1+k_{2} y}-\beta x_{1}-x_{2}-\frac{\mu_{0}}{1+k_{2} n} y\right),
\end{aligned}
$$




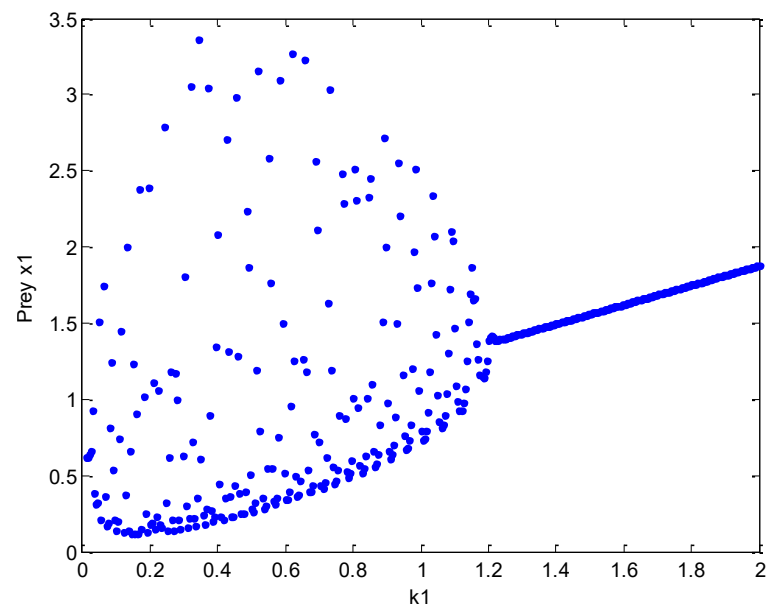

(a)

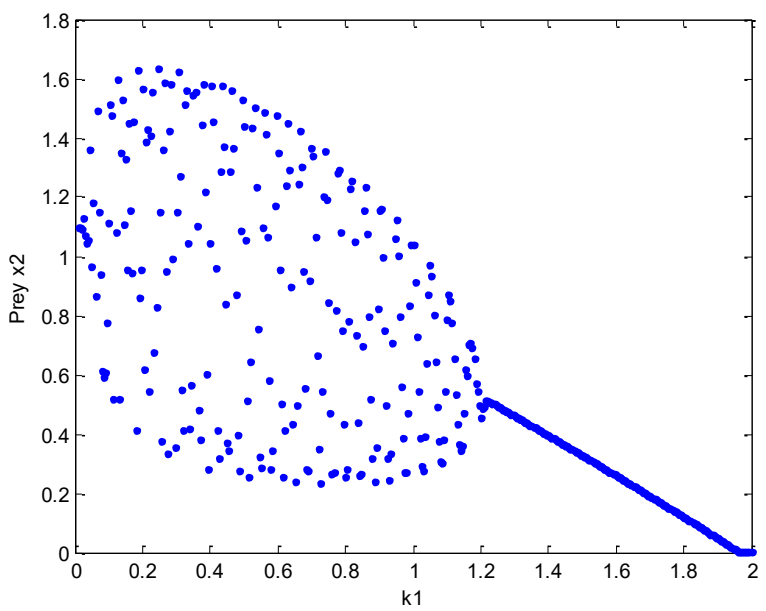

(b)

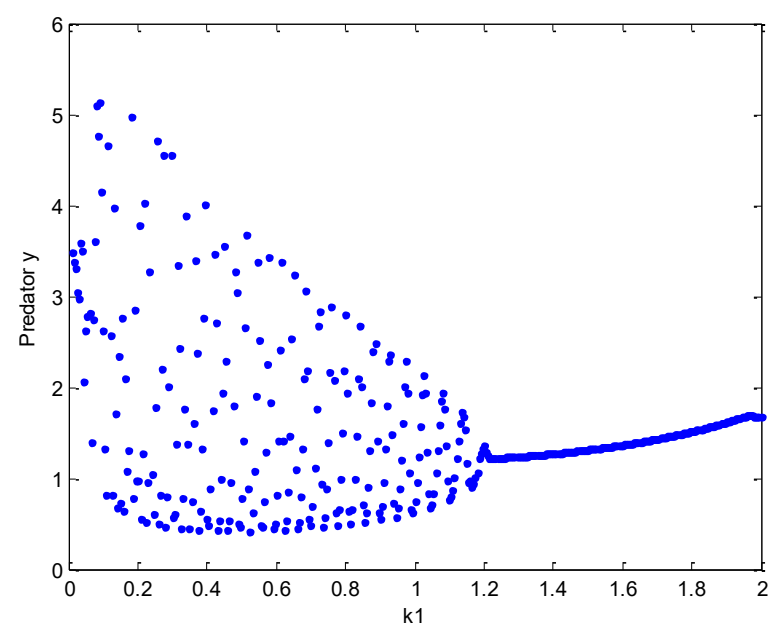

(c)

Figure 4. Bifurcation diagram for prey $x_{1}, x_{2}$, and predator $y$ with respect to the parameter $k_{1}$ when $k_{2}=0.04$ and other parameter values are given in (12)

$$
\frac{d y}{d t}=y\left(-d+\frac{c_{1} \varepsilon_{0}}{1+k_{1} m} x_{1}+\frac{c_{2} \mu_{0}}{1+k_{2} n} x_{2}-h y\right)
$$

First we investigate the effect of fear on the dynamics of system (11). So it is reasonable to study the system (11) without fear effect (i.e., $k_{i}=0, i=1,2$ ). We choose the other parameter value as

$$
r_{1}=12, r_{2}=2, \alpha=5, \beta=1, \varepsilon_{0}=1.6, \mu_{0}=0.01, m=1, n=1, c_{1}=1, c_{2}=1, d=1, h=0.001 .
$$

Figure 1 shows the phase diagram of system (11) for $k_{i}=0, i=1,2$. In absence of fear, oscillatory behaviour is observed. We now increase the value of $k_{2}$ from 0 to 0.04 , keeping $k_{1}=0$ fixed. Stable behaviour is observed for system (11) and the solutions converge to the coexistence equilibrium point $E^{*}=(0.6177,1.0891,3.6949)$ (see Figure 2). Now we increase the value of $k_{1}$ from 0 to 0.05 , a Hopf bifurcating periodic solution appears around the steady state $E^{*}=(1.5347,0.9467,2.7008)$ (see Figure 3). Bifurcation diagram with respect to the parameter $k_{1}$ is depicted in Figure 4. From Figures 3 and 4, we note that the increase amount of predator fear stabilizes the system. Taking the value of parameter $h=0.1$ and all other parameters are same as in Figure 3 , we observe that the solutions of system (11) converge to the equilibrium point $E^{*}=(0.8700,0.8608,3.3500)($ see Figure 5). Furthermore, from Figure 5, we observed that the increase amount of intraspecific competition within the predator population can induces stability of the system.

\section{Discussion}

In predator-prey interaction, predation is considered to be the main force that promotes coexistence of competing species by reducing the strength of competition [8]. If the predator chooses strongest competitor species, mostly 


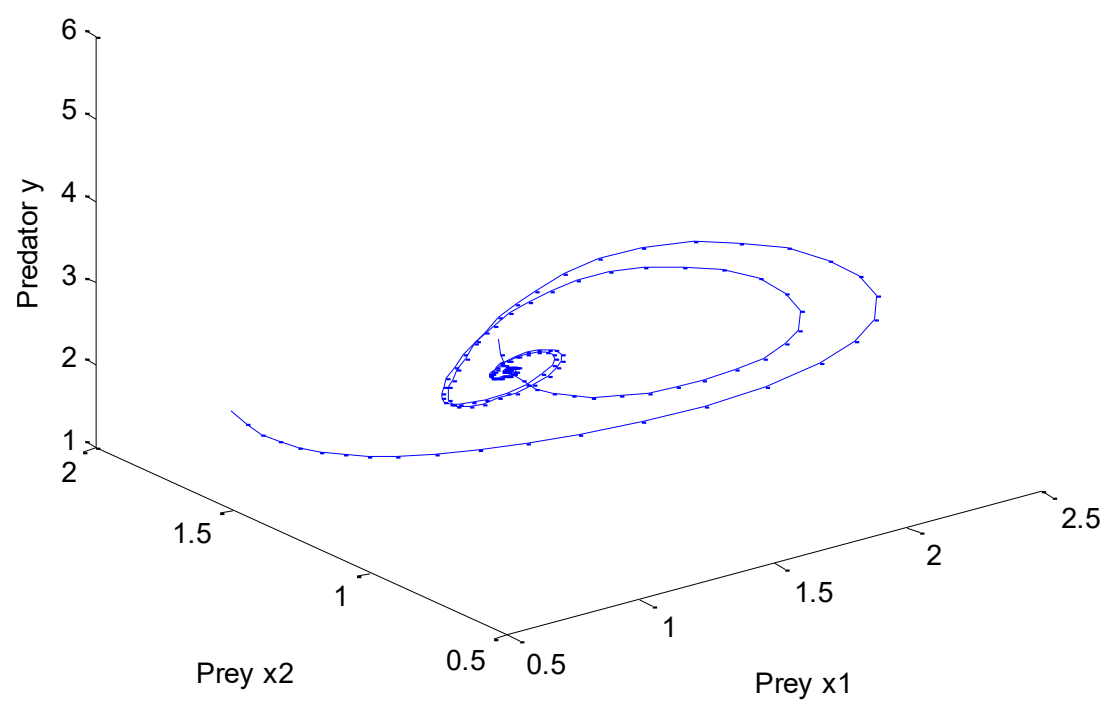

Figure 5. Phase portrait of system (11) for with parameters values $r_{1}=12, r_{2}=2, \alpha=5, \beta=1, \varepsilon_{0}=1.6, \mu_{0}=$ $0.01, c_{1}=1, c_{2}=1, d=1, h=0.1, k_{1}=0.05, k_{2}=0.04$ showing local stability of the equilibrium point $E^{*}=(0.8700,0.8608,3.3500)$

then it relives competition pressure on other species, thereby allowing coexistence of multiple species. Recent field experiments showed that predators can induce a non-consumptive effect on their prey, for example fear [30]. Due to predation fear, prey can adopt defensive strategies that disrupt coexistence [31]. To address fear induced coexistence on competing species, we developed a mathematical model of two competing prey species and one predator where predator not only kill both the prey but also shows non-consumptive effect upon them. Our system also includes intraspecific competition within the predator population. Takeuchi and Adachi [23] addresses an ecological system with the same type of species, but no fear effect, nor intraspecific competition within the predator populations obtaining coexistence results. The proposed model is biologically meaningful in the sense that any positive solution initiating in the positive orthant remains both non-negative and bounded.

Mathematical analysis of the model established that the system cannot collapse for any parameter value as the origin is always unstable. If the second prey has low intrinsic growth rate and the predator has a high death rate then the predator cannot prevent the first prey and tends to its carrying capacity; $E_{1}$ is an attractor whereas the opposite holds if the first prey has low intrinsic growth rate. If the intraspecific competition if stronger than the interspecific competition and the predator has the highest death rate then both the prey can coexist at $E_{12}$ while predator population goes into extinction due to large death rate. The first prey and the predator can coexist at $E_{13}$ when the second prey has moderate intrinsic growth rate. Again the second prey and the predator can coexist at $E_{23}$ as long as the intrinsic growth rate remains below a certain threshold value. Using invasion analysis, we derived criterion for uniform persistence of our model system that ensures the existence of positive (coexistence) equilibrium point. Local stability of the coexistence equilibrium point is possible if the ratio of intake capacity by the predator lie within an interval. The existence of Hopf bifurcation is shown by considering the level of fear as bifurcation parameter. The nature of limit cycle emerging through a Hopf bifurcation is predicted by calculating the coefficient of curvature of the limit cycle. In this paper we have not considered intraspecific competition rate $h$ as bifurcation parameter. But one obtain bifurcation result for taking $h$ as bifurcation parameter. When most of the predators are involved in intraspecific competition, stable coexistence increases (see Figure 5).

The novelty of our work is the inclusion of fear effect and intraspecific competition within the predator populations which are not considered in [23]. This investigation generalizes the existing knowledge of fear effect of predator on a single prey species $[15,16,18,19,21,22]$. Furthermore, previous studies ignore the fear effect on predation rate for obtaining coexistence results. As high level of fear can destroy coexistence that agrees with [31] still coexistence of predator and competing prey is possible with the increase of intraspecific competition within the predator population. Our theoretical observations will be helpful to verify some experimental data set of two competing prey and one predator system.

It may also be worthwhile to see how the other response function rather than Holling type I affects the dynamics 
of the system. From experimental observation, we have considered the fear effect on reproduction term of prey populations and predation rate still it is reasonable to see the fear effect on intraspecific, interspecific competition or death rate of prey populations.

\section{Conflict of interest}

The author declares that there is no conflict of interest in publishing this paper.

\section{Funding}

This research received no specific grant from any funding agency in the public, commercial or not-for-profit sectors.

\section{Acknowledgment}

The author is grateful to the anonymous reviewer for his/her helpful comments in improving the paper.

\section{References}

[1] R. D. Holt, "Predation, apparent competition, and the structure of prey communities," Theoretical Population Biology, vol. 12, no. 2, pp. 197-229, 1977.

[2] _ , "Spatial heterogeneity, indirect interactions, and the coexistence of prey species," The American Naturalist, vol. 124, no. 3, pp. 377-406, 1984.

[3] B. P. Kotler and R. D. Holt, "Predation and competition: The interaction of two types of species interactions," Oikos, vol. 54, no. 2, p. 256, 1989

[4] R. R. Vance, "Predation and resource partitioning in one predator - two prey model communities," The American Naturalist, vol. 112, no. 987, pp. 797-813, 1978.

[5] P. Chesson and J. J. Kuang, "The interaction between predation and competition," Nature, vol. 456, no. 7219, pp. 235-238, 2008.

[6] V. Křivan, “Competitive co-existence caused by adaptive predators," Evolutionary Ecology Research, vol. 5, no. 8, pp. 1163-1182, 2003.

[7] J. J. Kuang and P. Chesson, "Interacting coexistence mechanisms in annual plant communities: Frequency-dependent predation and the storage effect," Theoretical Population Biology, vol. 77, no. 1, pp. 56-70, 2010.

[8] J. Gurevitch, J. A. Morrison, and L. V. Hedges, "The interaction between competition and predation: A meta-analysis of field experiments," The American Naturalist, vol. 155, no. 4, pp. 435-453, 2000.

[9] S. L. Lima, "Nonlethal effects in the ecology of predator-prey interactions," BioScience, vol. 48, no. 1, pp. 25-34, 1998.

[10] S. Creel, D. Christianson, S. Liley, and J. A. Winnie, "Predation risk affects reproductive physiology and demography of elk," Science, vol. 315, no. 5814, pp. 960-960, 2007.

[11] S. Creel and D. Christianson, "Relationships between direct predation and risk effects," Trends in Ecology E Evolution, vol. 23, no. 4, pp. 194-201, 2008.

[12] W. Cresswell, "Non-lethal effects of predation in birds," Ibis, vol. 150, no. 1, pp. 3-17, 2008.

[13] _ "Predation in bird populations," Journal of Ornithology, vol. 152, no. S1, pp. 251-263, 2011.

[14] L. Y. Zanette, A. F. White, M. C. Allen, and M. Clinchy, "Perceived predation risk reduces the number of offspring Songbirds produce per year," Science, vol. 334, no. 6061, pp. 1398-1401, 2011.

[15] X. Wang, L. Zanette, and X. Zou, "Modelling the fear effect in predator-prey interactions," Journal of Mathematical Biology, vol. 73, no. 5, pp. 1179-1204, 2016.

[16] X. Wang and X. Zou, "Modeling the fear effect in predator-prey interactions with adaptive avoidance of predators," Bulletin of Mathematical Biology, vol. 79, no. 6, pp. 1325-1359, 2017.

[17] S. K. Sasmal and Y. Takeuchi, "Dynamics of a predator-prey system with fear and group defense," Journal of Mathematical Analysis and Applications, vol. 481, no. 1, p. 123471, 2020.

[18] S. Mondal, A. Maiti, and G. P. Samanta, "Effects of fear and additional food in a delayed predator-prey model," Biophysical Reviews and Letters, vol. 13, no. 04, pp. 157-177, 2018.

[19] H. Zhang, Y. Cai, S. Fu, and W. Wang, "Impact of the fear effect in a prey-predator model incorporating a prey refuge," Applied Mathematics and Computation, vol. 356, pp. 328-337, 2019.

[20] P. Panday, N. Pal, S. Samanta, and J. Chattopadhyay, "Stability and bifurcation analysis of a three-species food chain model with fear," International Journal of Bifurcation and Chaos, vol. 28, no. 01, p. 1850009, 2018.

[21] S. Pal, S. Majhi, S. Mandal, and N. Pal, "Role of fear in a predator-prey model with Beddington-DeAngelis functional response," Zeitschrift für Naturforschung A, vol. 74, no. 7, pp. 581-595, 2019.

[22] D. Mukherjee, "Study of fear mechanism in predator-prey system in the presence of competitor for the prey," Ecological Genetics and Genomics, vol. 15, p. 100052, 2020.

[23] Y. Takeuchi and N. Adachi, "Existence and bifurcation of stable equilibrium in two-prey, one-predator communities," Bulletin of Mathematical Biology, vol. 45, no. 6, pp. 877-900, 1983. 
[24] G. Birkhoff and G.-C. Rota, Ordinary differential equation. Boston: Ginn and Co., 1982.

[25] V. Hutson, "The existence of an equilibrium for permanent systems," The Rocky Mountain Journal of Mathematics, vol. 20, no. 4, pp. 1033-1040, 1990.

[26] _ _ "A theorem on average Liapunov functions," Monatshefte für Mathematik, vol. 98, no. 4, pp. 267-275, 1984.

[27] Z. Qiu, "Dynamics of a model for virulent phage T4," Journal of Biological Systems, vol. 16, no. 04, pp. 597-611, 2008.

[28] W. Liu, "Criterion of Hopf bifurcations without using eigenvalues," Journal of Mathematical Analysis and Applications, vol. 182, no. 1, pp. 250-256, 1994.

[29] X. Wang, L. Deng, and W. Zhang, "Hopf bifurcation analysis and amplitude control of the modified Lorenz system," Applied Mathematics and Computation, vol. 225, pp. 333-344, 2013.

[30] O. J. Schmitz, "Predators affect competitors' coexistence through fear effects," Nature, vol. 570, no. 7759, pp. 43-44, 2019.

[31] R. M. Pringle et.al, "Predator-induced collapse of niche structure and species coexistence," Nature, vol. 570, no. 7759, pp. 58-64, 2019.

(c)2021 by the Author(s). This article is an open access article distributed under the terms and conditions of the Creative Commons Attribution-NonComercial 4.0 International License. Editorial of JJBM: Department of Mathematics, State University of Gorontalo, Jln. Prof. Dr. Ing. B. J. Habibie, Bone Bolango 96119, Indonesia. 


\section{Submit your manuscript at http://ejurnal.ung.ac.id/}

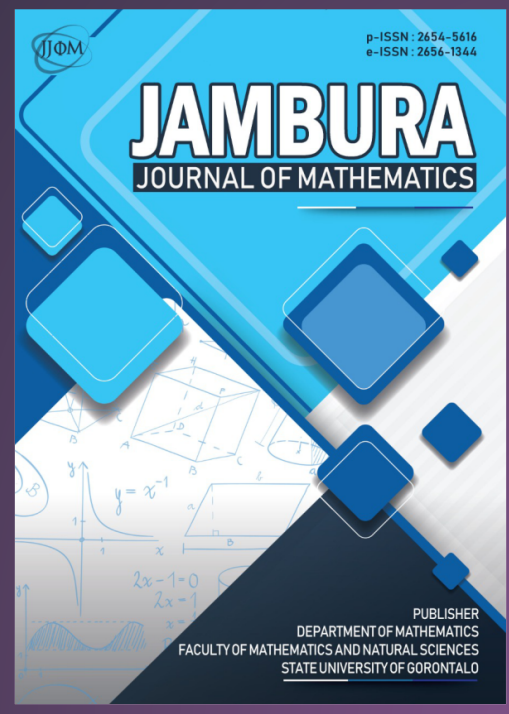

Jambura Journal of Mathematics

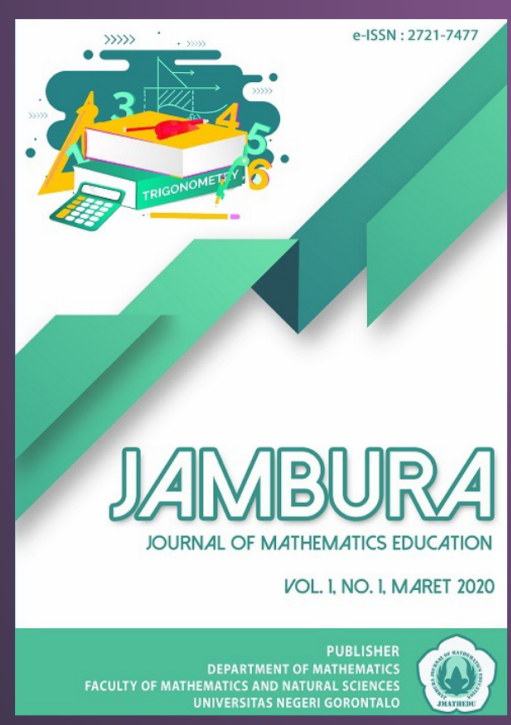

Jambura Journal of Mathematics Education
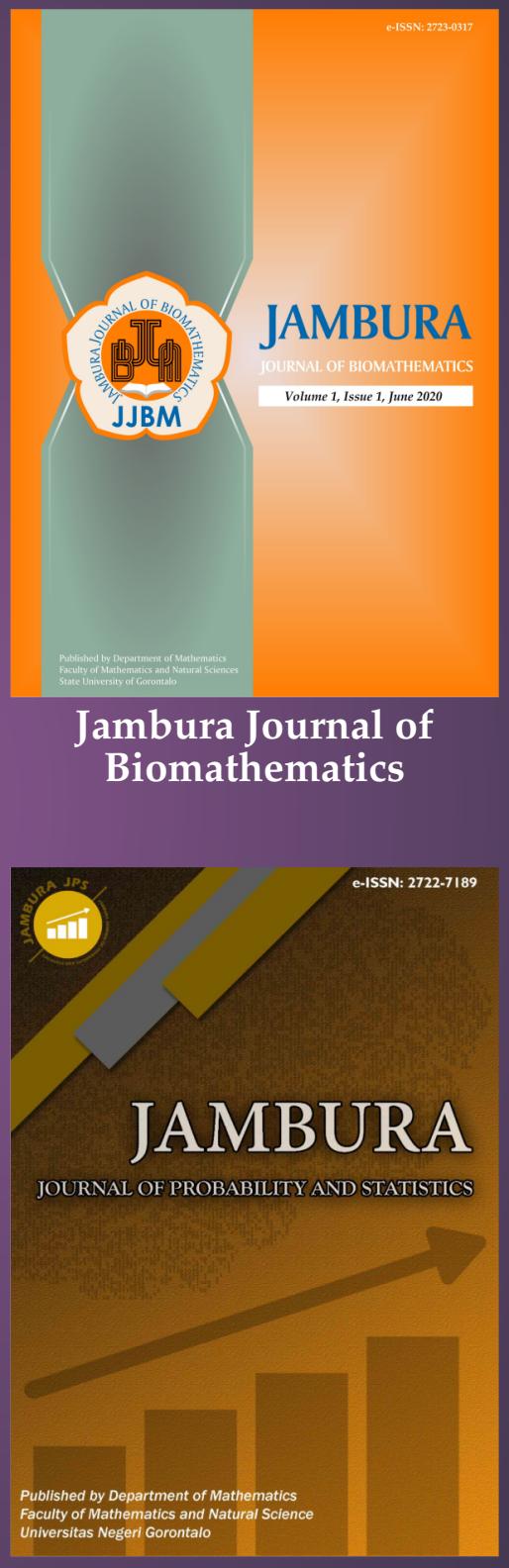

Jambura Journal of Probability and Statistics

Published by

Department of Mathematics

Faculty of Mathematics and Natural Sciences

State University of Gorontalo 